\title{
Fine-tune of intrinsic ERK activity by extrinsic BMP signaling in mouse embryonic stem cells
}

\author{
Zhongwei Li, Ye-Guang Chen
}

The State Key Laboratory of Biomembrane and Membrane Biotechnology, Tsinghua-Peking Center for Life Sciences, School of Life Sciences, Tsinghua University, Beijing 100084, China

$\triangle$ Correspondence: ygchen@tsinghua.edu.cn

Received March 18, 2012 Accepted March 26, 2012

Embryonic stem (ES) cells hold great promise in regenerative medicine and it is an urgent task to understand the underlying molecular mechanisms that control ES cell fate choice between self-renewal and differentiation. In mouse ES cells, extrinsic leukemia inhibitory factor (LIF) and bone morphogenetic protein (BMP) signaling pathways play pivotal roles in maintaining the self-renewal status under serum and feeder free culture conditions. Intrinsic extracellular-signal regulated kinase (ERK) activity is also important in determining mouse ES cell fate-low ERK activity keeps mouse ES cell self-renewal while high ERK activity drives differentiation. We recently found that while LIF signaling augments ERK activity, BMP signaling inhibits ERK activity in mouse ES cells via direct upregulation of an ERK phosphatase-dual-specificity phosphatase 9. The cooperative effects of LIF and BMP signaling keep appropriate ERK activity and maintain mouse ES cell self-renewal ( $L i$ et al., 2012). These findings shed light on how extrinsic signals converge to intrinsic signaling molecules to regulate cell fate determination. This perspective summarizes our recent new findings and discusses the current unsolved questions and future directions.

Embryonic stem (ES) cells undergo self-renewal while maintain pluripotency in appropriate culture conditions (Smith, 2001). Directed differentiation of embryonic stem cells to specific cell lineages holds great promise in regenerative medicine (Passier et al., 2008). Therefore, it is critical to understand the underlying molecular mechanisms that govern ES cell fate determination. It has been shown that the maintenance of ES cell self-renewal requires delicate regulation in different levels (Pera and Tam, 2010; Orkin and Hochedlinger,
2011; Young, 2011). Extrinsically, leukemia inhibitory factor (LIF) and bone morphogenetic protein (BMP) signaling have been shown to be sufficient in supporting the self-renewal of mouse ES cells in the absence of serum and feeder cells (Ying et al., 2003a). Intrinsically, core transcription factors have been shown to be critical in establishing a transcriptional network in which pluripotency-related genes are expressed while differentiation-related genes are repressed in a poised-state (Boyer et al., 2005; Young, 2011). Extracellular-signal regulated kinase (ERK) is one of the most important intrinsic factors. ERK is an intracellular mediator of the mitogen-activated protein kinase (MAPK) signaling pathways and plays key roles in growth and differentiation in various cell types (Kolch, 2005). High ERK activity as induced by forced expression of constitutively active RAS leads to primitive endoderm differentiation of mouse ES cells (Yoshida-Koide et al., 2004), while inhibition of ERK activity makes mouse ES cell refractory to differentiation cues (Kunath et al., 2007). The fact that maintenance of mouse ES cells can be achieved solely by chemical inhibitors which keep low ERK and GSK3 activity (the so-called "2i" or "3i" culture conditions) strongly indicates the importance of ERK activity in mouse ES cell self-renewal (Ying et al., 2008). As a delicate biological system, how extrinsic signaling pathways and intrinsic ERK activity are linked together remains obscure, and it seems like a paradox that extrinsic LIF signaling was found to enhance ERK activity which would lead to instability of self-renewal status (Burdon et al., 1999; Ying et al., 2003a). In our recent study, we found that BMP signaling, via direct upregulation of dual-specificity phosphatase 9 (DUSP9), represses ERK activity, thus maintaining mouse ES cells in a stable self-renewal status in the presence of LIF ( $\mathrm{Li}$ et al., 2012).

Considering the importance of BMP signaling and ERK 
activity in ES cell biology, the link between BMP signaling and ERK activity in mouse ES cells was previously investigated by several groups. Ying et al. found that short BMP4 treatment for 15 min or 1 hour activates ERK activity (Ying et al., 2003a). Qi et al. found the inhibitory effect of BMP4 on ERK activity upon $5 \mathrm{~min}$ treatment and observed the recovery of ERK activity within 1 hour (Qi et al., 2004). A recent work from Zhang et al. also showed that BMP4 inhibits ERK activity within a short time (10 or $30 \mathrm{~min}$ ), but ERK activity recovers within 1 hour (Zhang et al., 2010). As BMP4 and LIF are constitutively included in the culture medium to maintain mouse ES cell self-renewal, we, thus, speculated that it is important to follow ERK activity changes upon BMP4 treatment in a longer period. Consistent with Ying et al. (2003a), our data revealed that BMP4 treatment enhances ERK activity within 4 hours but leads to a decrease of ERK activity after longer treatment time ( 8 hours and beyond). This effect is independent of LIF or FGF signaling (Li et al., 2012).

Then, we attempted to identify the mechanism underlying the BMP-mediated inhibition of ERK activity. The relatively slow inhibitory effect of BMP4 led us to test whether this effect was indirect. Indeed, blockage of de novo protein synthesis by cycloheximide $(\mathrm{CHX})$ abolished the inhibitory effect of BMP4 on ERK activity, indicating that this effect involves novel transcription and translation. Our previous chromatin immunoprecipitation (ChIP)-seq and microarray data suggested that dual-specificity phosphatase 9 (DUSP9) could be the target as its expression was upregulated by BMP4 and its promoter was shown to be bound by both Smad1/5 and Smad4. Importantly, it was previously established that DUSP9 could inactivate ERK activity through direct dephosphorylation of ERK (Owens and Keyse, 2007). Upregulation of DUSP 9 by BMP4 was validated at both MRNA and protein levels. Among a cohort of the 11 DUSP family members examined, it was the only phosphatase that was upregulated by BMP signaling. These results suggested that DUSP9 may mediate BMP repression of ERK activity in mouse ES cells.

We then set out to test this assumption. Overexpression of DUSP9 inhibited ERK activity in a dose-dependent manner and ERK target gene expression. By using a dominant-negative form of DUSP9 (dn-DUSP9) which blocked the activity of endogenous DUSP9, we found that BMP signaling no longer repressed ERK activity. Similarly, when DUSP9 expression was knocked down by siRNA, BMP inhibition of ERK activity was abolished. Thus, DUSP9 mediates BMP inhibition of ERK activity in mouse ES cells.

BMP signaling exerts its function by binding to its membrane receptors to activate the intracellular Smad1/5/8 proteins (Massagué and Chen, 2000; Feng and Derynck, 2005). Activation of Smad1/5/8 by receptor-mediated phosphorylation promotes their interaction with Smad4 and translocation of this Smad complex from the cytoplasm to the nucleus, where they function as transcription factors to regulate gene expression. We assumed that Dusp9 may be regulated in this way. Knockdown of Smad4 in mouse ES cells reduced the induction of Dusp9 by BMP4, and importantly, ERK activity was no longer repressed in the absence of Smad4. Consistently, a dramatic decrease of DUSP9 expression was observed in 3.5 day Smad4 knockout mouse embryos. Similar results were observed when the expression of Smad1 and Smad5 was depleted by knockdown and knockout. Promoter analysis confirmed that DUSP9 is a BMP target-the Dusp9 promoter responded to BMP signaling well. By using a series of promoter truncations, we identified the minimal responsive promoter fragment. A single nucleotide mutation in the putative Smad binding element (SBE) in this fragment abolished BMP responsiveness. ChIP assay also confirmed the binding of Smad1/5 and Smad4 to Dusp9 promoter that contains the SBE upon BMP4 treatment.

We then determined the importance of Dusp9 in mediating BMP functions. Forced expression of DUSP9, mimicking the MEK inhibitor PD184352, partially bypassed the requirement of BMP4 in sustaining self-renewal of mouse ES cells in cooperation with LIF. Moreover, inhibition of DUSP9 activity by dn-DUSP9 or DUSP9 knockdown reduced the expression of the pluripotency-related genes Nanog and Rex1 even in the presence of BMP4 and LIF. These results indicate that induction of Dusp9 is necessary for BMP signaling to maintain self-renewal in cooperation with LIF. In the absence of exogenous growth factors, mouse ES cells will go spontaneous neural differentiation and BMP signaling could significantly inhibit this process (Ying et al., 2003b; Fei et al., 2010). We found that overexpression of DUSP9 mimicked BMP4 and the MEK inhibitor PD184352 in repressing neural differentiation and on the contrary, expression of dn-DUSP9 or knockdown of DUSP9 impaired the neural inhibitory effects of BMP4. These results highlighted the importance of Dusp9 in mediating neural inhibition function of BMP4.

BMP signaling regulates a large number of target genes in mouse ES cells, and our early investigation indicated that inhibition of development/differentiation genes by BMP is important for BMP to maintain mouse ES cell pluripotency (Fei et al., 2010). Inhibitors of differentiation (Id) family proteins were reported to be the important BMP targets that promote mouse ES cell self-renewal by inhibiting differentiation to the neural lineage (Ying et al., 2003a). BMP has been shown to inhibit ERK activity in mouse ES cells in short treatment time (Qi et al., 2004; Zhang et al., 2010). This study identified Dusp9 as the linker that mediates extrinsic BMP4 signaling to inhibit intrinsic ERK activity in a long and steady way. This study extended the mechanism of how BMP4 contributes to mouse ES cell self-renewal maintenance and revealed the delicate balancing of intrinsic ERK activity by the extrinsic signals BMP and LIF (as summarized in Fig. 1). Several interesting phenomena were also observed during the study which are worth further investigation. We found that the BMP/DUSP9/ERK cascade exists only in mouse embryonic stem cells, but not in other tested mouse somatic cells. 


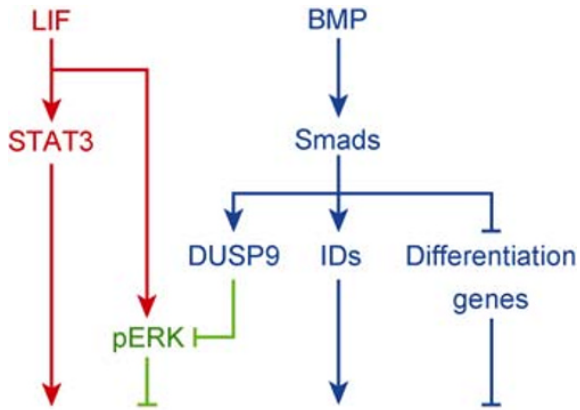

Mouse embryonic stem cells self-renewal

Figure 1. Maintenance of mESC self-renewal by LIF and BMP signaling. STAT3 activated by LIF signaling and Id family proteins activated by BMP/Smad signaling contribute to self-renewal maintenance. BMP can also promote mESC self-renewal by inhibiting differentiation gene expression. The differentiation promoting signal p-ERK activated by LIF signaling is attenuated by BMP signaling via Smad-mediated upregulation of DUSP9. The balanced p-ERK activity ensures mESC self-renewal maintenance. mESC, mouse embryonic stem cess; LIF, leukemia inhibitory factor; BMP, bone morphogenetic protein; STAT3, signal transducer and activator of transcription protein 3; ERK, extracellular signal regulated kinase; DUSP9, dual-specificity phosphatase 9; IDs, inhibitors of differentiation.

The ES-specific regulation of DUSP9 by BMP signaling may be due to the cooperative transcriptional activation between ES-specific factors and Smad proteins, such as the recently identified stem cell coactivator (SCC) complex (Fong et al., 2011). It will be interesting to test this possibility. We also found that in 3.5 day Smad4 knockout mouse embryos, DUSP9 was dramatically reduced compared to wild-type embryos. It is unclear what roles the Smad4-dependent DUSP9 expression plays in mouse early development. Smad4 knockout mice exhibited severe gastrulation defect which could be rescued by tetraploid complementation assay (Sirard et al., 1998; Yang et al., 1998), indicating the repaired function of extraembryonic tissues. Further investigation indicated that Smad4 is required for appropriate visceral endoderm development (Sirard et al., 1998). Consistent with this, Smad5 knockout mice also showed extraembryonic defects in later developmental stages (Chang et al., 1999). Importantly, Dusp9 knockout mice died due to failure of labyrinth development in the placenta (Christie et al., 2005). Thus, DUSP9 is likely to mediate the proper extraembryonic development function of BMP/Smad signaling, which warrants further study. We also found that BMP signaling inhibits ERK activity in human ES cells and human DUSP9 is upregulated by BMP signaling. Thus, it looks like the BMP/DUSP9/ ERK cascade is conserved in human ES cells. Considering the nonexistence of this cascade in mouse somatic cells, we reason that it may only exist in "stemness" cells. It merits future examination of whether it is present in mouse EpiSCs, tissue or cancer stem cells and what the significance of this regulation is under different contexts.

\section{ACKNOWLEDGEMENTS}

This work was supported by grants from the State Key Development Program for Basic Research of China (973 Program) (Grant Nos. 2010CB833706 and 2011CB943803), the National Natural Science Foundation of China (Grant Nos. 30930050 and 30921004 ).

\section{REFERENCES}

Boyer, L.A., Lee, T.I., Cole, M.F., Johnstone, S.E., Levine, S.S., Zucker, J.P., Guenther, M.G., Kumar, R.M., Murray, H.L., Jenner, R.G., et al. (2005). Core transcriptional regulatory circuitry in human embryonic stem cells. Cell 122, 947-956.

Burdon, T., Stracey, C., Chambers, I., Nichols, J., and Smith, A. (1999). Suppression of SHP-2 and ERK signalling promotes self-renewal of mouse embryonic stem cells. Dev Biol 210, 30-43.

Chang, H., Huylebroeck, D., Verschueren, K., Guo, Q., Matzuk, M.M., and Zwijsen, A. (1999). Smad5 knockout mice die at mid-gestation due to multiple embryonic and extraembryonic defects. Development 126, 1631-1642.

Christie, G.R., Williams, D.J., Macisaac, F., Dickinson, R.J., Rosewell, I., and Keyse, S.M. (2005). The dual-specificity protein phosphatase DUSP9/MKP-4 is essential for placental function but is not required for normal embryonic development. Mol Cell Biol 25, 8323-8333.

Fei, T., Xia, K., Li, Z., Zhou, B., Zhu, S., Chen, H., Zhang, J., Chen, Z., Xiao, H., Han, J.D., et al. (2010). Genome-wide mapping of SMAD target genes reveals the role of BMP signaling in embryonic stem cell fate determination. Genome Res 20, 36-44.

Feng, X.H., and Derynck, R. (2005). Specificity and versatility in tgf-beta signaling through Smads. Annu Rev Cell Dev Biol 21, 659-693.

Fong, Y.W., Inouye, C., Yamaguchi, T., Cattoglio, C., Grubisic, I., and Tjian, R. (2011). A DNA repair complex functions as an Oct4/Sox2 coactivator in embryonic stem cells. Cell 147, 120-131.

Kolch, W. (2005). Coordinating ERK/MAPK signalling through scaffolds and inhibitors. Nat Rev Mol Cell Biol 6, 827-837.

Kunath, T., Saba-El-Leil, M.K., Almousailleakh, M., Wray, J., Meloche, S., and Smith, A. (2007). FGF stimulation of the Erk1/2 signalling cascade triggers transition of pluripotent embryonic stem cells from self-renewal to lineage commitment. Development 134 , 2895-2902.

Li, Z., Fei, T., Zhang, J., Zhu, G., Wang, L., Lu, D., Chi, X., Teng, Y., Hou, N., Yang, X., et al. (2012). BMP4 Signaling Acts via dual-specificity phosphatase 9 to control ERK activity in mouse embryonic stem cells. Cell Stem Cell 10, 171-182.

Massagué, J., and Chen, Y.G. (2000). Controlling TGF-beta signaling. Genes Dev 14, 627-644.

Orkin, S.H., and Hochedlinger, K. (2011). Chromatin connections to pluripotency and cellular reprogramming. Cell 145, 835-850.

Owens, D.M., and Keyse, S.M. (2007). Differential regulation of MAP kinase signalling by dual-specificity protein phosphatases. Oncogene $26,3203-3213$. 
Passier, R., van Laake, L.W., and Mummery, C.L. (2008). Stem-cellbased therapy and lessons from the heart. Nature 453, 322-329.

Pera, M.F., and Tam, P.P. (2010). Extrinsic regulation of pluripotent stem cells. Nature 465, 713-720.

Qi, X., Li, T.G., Hao, J., Hu, J., Wang, J., Simmons, H., Miura, S., Mishina, Y., and Zhao, G.Q. (2004). BMP4 supports self-renewal of embryonic stem cells by inhibiting mitogen-activated protein kinase pathways. Proc Natl Acad Sci U S A 101, 6027-6032.

Sirard, C., de la Pompa, J.L., Elia, A., Itie, A., Mirtsos, C., Cheung, A., Hahn, S., Wakeham, A., Schwartz, L., Kern, S.E., et al. (1998). The tumor suppressor gene Smad4/Dpc4 is required for gastrulation and later for anterior development of the mouse embryo. Genes Dev 12, 107-119.

Smith, A.G. (2001). Embryo-derived stem cells: of mice and men. Annu Rev Cell Dev Biol 17, 435-462.

Yang, X., Li, C., Xu, X., and Deng, C. (1998). The tumor suppressor SMAD4/DPC4 is essential for epiblast proliferation and mesoderm induction in mice. Proc Natl Acad Sci U S A 95, 3667-3672.

Ying, Q.L., Nichols, J., Chambers, I., and Smith, A. (2003a). BMP induction of Id proteins suppresses differentiation and sustains embryonic stem cell self-renewal in collaboration with STAT3. Cell 115, 281-292.

Ying, Q.L., Stavridis, M., Griffiths, D., Li, M., and Smith, A. (2003b). Conversion of embryonic stem cells into neuroectodermal precursors in adherent monoculture. Nat Biotechnol 21, 183-186.

Ying, Q.L., Wray, J., Nichols, J., Batlle-Morera, L., Doble, B., Woodgett, J., Cohen, P., and Smith, A. (2008). The ground state of embryonic stem cell self-renewal. Nature 453, 519-523.

Yoshida-Koide, U., Matsuda, T., Saikawa, K., Nakanuma, Y., Yokota, T., Asashima, M., and Koide, H. (2004). Involvement of Ras in extraembryonic endoderm differentiation of embryonic stem cells. Biochem Biophys Res Commun 313, 475-481.

Young, R.A. (2011). Control of the embryonic stem cell state. Cell 144, 940-954.

Zhang, K., Li, L., Huang, C., Shen, C., Tan, F., Xia, C., Liu, P., Rossant, J., and Jing, N. (2010). Distinct functions of BMP4 during different stages of mouse ES cell neural commitment. Development 137, 2095-2105. 